\title{
Total Knee Replacement in Tuberculous Arthritis - Reporting an Unsuspected Case
}

\author{
Dr. P. Ramesh Vyravan, Dr.B. Mohan Choudhary, Dr. M. Mohan Kumar, \\ Sri Ramachandra Medical College, Chennai
}

Total knee arthroplasty is done to reduce pain and disability in elderly individuals with conditions like osteoarthritis and rheumatoid arthritis. One of the dreaded complications of TKR is infection which causes surgery a total failure and furthermore the subsequent treatment becomes more complicated and causes more morbidity and financial expenditure to the patient. TKR to treat active joint tuberculosis remains controversial and most authors advice not to do joint replacements in cases with active discharging sinuses ${ }^{1,4,9-11}$. As a precaution it is always necessary to rule out infection pre operatively by thoroughly investigating the patient. But sometimes we can encounter unexpected things.

A 55 year old female who was apparently normal two years back developed right knee pain. Pain is insidious, continuous, not associated with fever or any trauma. She is not a known case of TB of any other organ. But she is a known case of rheumatoid arthritis and epilepsy, on treatment. On investigating she had increased ESR of 56 for one hour, CRP is elevated to 2.4 and serum alkaline phosphatase elevated to 155 .

As there were no signs to suspect active TB in any of the systems or organs both clinically and radiologically, we proceeded with the total knee replacement. But on opening the joint synovium looked inflamed and hence it was sent for biopsy and TKR was completed with Genesis II (Smith and Nephew). The biopsy report came as granulomatous lesion consistent with tuberculosis. Synovium specimen showed epithelioid granuloma with Langhans Giant cells. But AFB and fungal staining came as negative.

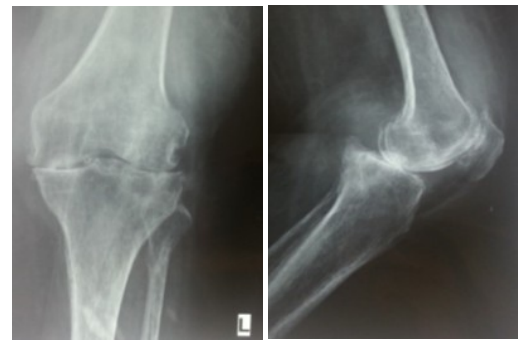

Fig 1 - Plain X - Ray of the left knee AP and Lateral views

Post operatively patient was started on Anti TB treatment. Patient was mobilized next day of surgery with active knee bending exercises and after one week patient was made to walk full weight bearing and patient had no pain. Sutures were removed fifteenth post op day and discharged. Patient had been followed up for six months so far without any reactivation of tuberculosis. Six months follow up post op x-ray did not show any osteolysis of both femoral and tibial components.

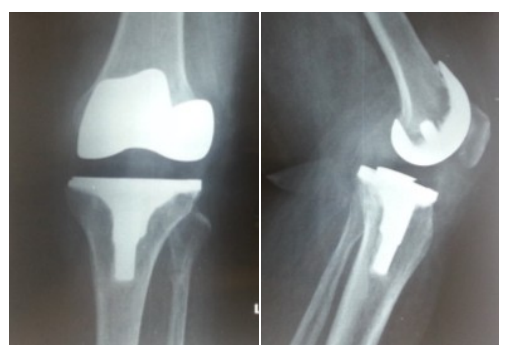

Fig 2 - Immediate post op Plain X - Ray of the left knee AP and Lateral views

Skeletal tuberculosis is a common infection in India which is around $2 \%$ of all musculo skeletal tuberculosis. In the musculo skeletal system it affects most commonly the spine and next in order is hips and knees. When literature was reviewed TKR has been advised for subclinical TB and there are reports that TKR reduces pain, restores excellent range of motion and even TB gets cured and reactivation of TB is less. But in 
active cases TKR should be avoided. Active cases are those who are proven TB with multi organ involvement, abnormal ESR, CRP and radiological investigations showing active lesions in the joint.

The treatment of choice for active TB in a joint includes debridement, arthrodesis and resection arthroplasty along with anti TB regimen. But debridement will damage the cartilage causing pain and reduced range of movement. Arthrodesis will give pain free but restricted joint movement. Resection arthroplasty will give hypermobile painless but unstable joint. Hence nowadays we are exploring to treat joint with active TB to reduce all these drawbacks and give the patient a better lifestyle.

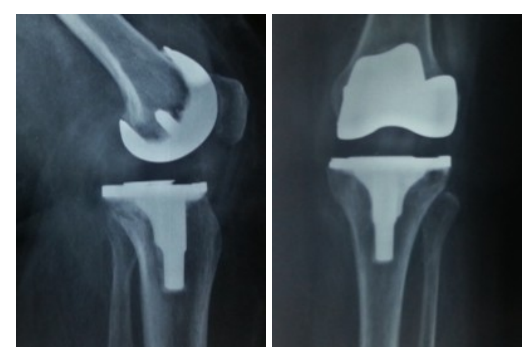

Figs 3 - Six months follow up X- Ray

TKR can be done in joints with active TB if the patient is properly treated with anti TB treatment pre operatively and postoperatively. Su et al ${ }^{2}$ reviewed 15 patients (16knees) who had TKR done. They were divided into two groups. Group I of 8 knees had active TB and treated pre operatively and post operatively with anti TB regimen. Group II of 8 knees included cases with no active TB hence no anti TB regimen given pre operatively but as they were diagnosed to have TB after surgery they were administered TB drugs post operatively. Group I had one relapse due to steroid intake. Group II had four relapses.

Sidhu et $\mathrm{al}^{3}$ reported 23 cases of active TB hip confirmed histopathologically and by PCR test. All were given three months pre op and 18 months post op TB treatment and treated with cemented THR. At an average follow up of 4.7 years there were no reactivations.

Yoon et $\mathrm{al}^{4}$ reported seven cases who had active TB with hip abscess but no discharging sinus. They were successfully treated with uncemented THR with thorough debridement and 12 months of post op TB drugs. At a mean follow up 4.8 years there was no relapse.

The management of reactivated infection in a patient with unsuspected $\mathrm{TB}^{1}$ before arthroplasty will be challenging. If the patient receives efficient anti TB regimen peri and post operatively the recurrence can be avoided. Also if wound debridement was given pre operatively causing bone defects then prosthetic fitting may become difficult. In that situations morselized autograft or iliac crest graft will be useful. If there is reactivation of TB then proper TB regimen with or without debridement alone can save the prosthesis.

If prosthesis cannot be saved then its removal with or without arthrodesis will be the choice. So in postoperative follow up if patient has signs of infection with culture showing negative the surgeons should be aware of possibility of TB in the joint. To achieve successful outcome we should plan proper pre, peri and postoperative TB care with or without debridement and during the procedure we should completely curette inflamed soft tissues and necrotic bone ${ }^{1}$. Pre op TB regimen should be for atleast two weeks so that inflammatory markers (ESR and CRP) $)^{1}$ show positive reaction and post-operative regimen should be for minimum of $12-15$ months $^{6}$.

Single stage joint replacement can be used to treat active joint $\mathrm{TB}^{1}$ for the following reasons,

1. M tuberculosis divides once in 15-20 hours which is slow when compared to S.Aureus which is $20 \mathrm{~min}$.

2. TB Bacilli form biofilm that differs from other pyogenic organisms and according to $\mathrm{Ha}$ et $\mathrm{al}^{5} \mathrm{~TB}$ bacilli rarely or don't adhere to the metal surface and rarely forms or don't form biofilm. Ma et al ${ }^{7}$ study found that no biofilm formation for $\mathrm{M}$ tuberculosis on the surface of cobalt-chromium-molybdenum alloy or titanium alloy and

3. Metal implants have been used successfully in TB Spondylitis patients. Hence with proper coverage primary joint replacement can be successfully done in active TB arthritis cases. 


\section{Bibliography}

[1]. Yi-chao Zhang, MD; Hong Zhang MD; One stage Total joint arthroplasty for patients with active tuberculosis.,healio.com, Vol 36 Issue 5, May 2013

[2]. Su JY, Huang TL, Lin SY, Total knee arthroplasty in tuberculous arthritis, CORR,1996 (323) 181-187

[3]. Sidhu S, Singh AP, Singh AP, Total hip replacement in active advanced tuberculous arthritis, JBJS Br 2009,(10) 1301-1304

[4]. Yoon TR, Rowe SM et al, Immediate Cementless THR for active TB Hip, J Arthroplasty 2005, 20(7); 923-6

[5]. Ha KY, Chang YG et al, Adherence and biofilm formation of S.Epidermidis, M tuberculosis on various spine implants, Spine ( Phila Pa 1976), 2005; 30(1): 38-43

[6]. Neogi DS, Yadav CS, Kumar A, THR in patients with active TB of hip with advanced arthritis, CORR, 2010; (468): 605-612

[7]. Total Knee Replacement in unsuspected tuberculosis, M. I. B Besser, British Medical Journal, 14 June 1980

[8]. Ma J, Li GQ, Cao L. Adhesive ability of Mycobacterium tuberculosis onto the surface of different joint prosthesis materials. Chin J Tissue Eng Res.2012; 16(47):8807-8812.

[9]. Hardinge K, Cleary J, Charnley J. Low-friction arthroplasty for healed septic and tuberculous arthritis. JBJS Br. 1979; 61:144-147.

[10]. Caparros AB, Sousa M, Ribera ZJ, Uceda CP, Moya CF. Total hip arthroplasty for tuberculous coxitis. IntOrthop.1999; 23(6):348350

[11]. Kim YY, Ko CU, Ahn JY, Yoon YS, Kwak BM. Charnley low-friction arthroplasty in tuberculosis of the hip. An8- to 13-year follow-up. JBJS Br.1988; 70(5):756-760 